\title{
Cell Death in MMTV-c-myc Transgenic Mouse Mammary Tumors May Not Be Typical Apoptosis
}

\author{
Dezhong Joshua Liao and Robert B. Dickson \\ Lombardi Cancer Center (DJL, RBD) and Departments of Oncology (RBD) and Cell Biology (RBD), Georgetown \\ University Medical Center, Washington, DC; and Department of Pathology (DJL), Karmanos Cancer Institute, Wayne \\ State University School of Medicine, Detroit, Michigan
}

SUMMARY: Enforced expression of c-myc has been shown to serve as an apoptotic stimulus in cultured cells. Prior studies have also demonstrated that several tissues expressing c-myc transgene display a large number of dead cells, although a morphologic or biochemical verification of apoptosis in these tissues has actually not been presented. In the present study, we examined the morphologic properties of cell death in the mammary tumors developed from MMTV-c-myc transgenic mice. We found that c-myc-expressing mammary tumor cells exhibited malformation of mitochondria, characterized by an amorphous matrix with very few cristae. The mitochondria were also frequently degenerated by lysis of the matrix and cristae. The protein level of cytochrome $\mathrm{c}$ was much lower in the areas of c-myc-expressing tumor cells compared with the adjacent tumor foci, which was previously shown to have decreased expression of c-myc, reduced frequencies of cell death, and increased frequencies of proliferating cells. In the c-myc-expressing tumor areas, there were many dying or dead cells organized in clusters, termed "dead cell islands." These cells exhibited shrinkage, DNA breakage as indicated by a positive TUNEL staining, and nuclear localization of apoptosis-inducing factor, but a lack of typical apoptotic morphology, such as nuclear condensation and formation of cell membrane blebs and apoptotic bodies. Many macrophages infiltrated into these dead cell islands, engulfing the dying or dead tumor cells. In the total tumor tissue, the protein level of caspase-3 was very low, and the poly(ADP)-ribose polymerase was present mainly as the unprocessed, inactive form. Collectively, these results suggest that programmed cell death in the c-myc transgenic mammary tumor tissue may not be typical apoptosis and may involve a caspase-independent mechanism. (Lab Invest 2003, 83:1437-1449).

$A$ review of the literature on the differences between apoptosis and other types of cell death, mainly necrosis, is currently very confusing, mainly because there is a lack of a strict definition of apoptosis (Afford and Randhawa, 2000; Columbano, 1995; Fiers et al, 1999; Johnson, 2000; Kroemer et al, 1998; Levin et al, 1999; Lockshin, 1999; Majno and Joris, 1999; Nicotera et al, 1999). As summarized by Blagosklonny (2000), apoptosis has been commonly described, but not precisely defined, from four different points of view: 1) programmed cell death; 2) a combination of morphologic features, such as nuclear fragmentation, chromatin condensation, DNA degradation, cell shrinkage, and membrane blebbing; 3) an active process of dying that requires energy and de novo gene expression; and 4) cell death that is nonnecrotic. The last point of view may be the main source of confusion because the term "necrosis" is

DOI: 10.1097/01.LAB.0000090153.13977.AE

Received February 11, 2003.

This work was supported by National Institues of Health grants ROI CA72460 and AG1496 (to RBD), by Department of Defense grant DAMD17-00-1-0270 (to DJL and RBD), and by a DOD postdoctoral fellowship (to DJL).

Address reprint requests to: Dr. Joshua Liao, HWCRC, Room 703, Karmanos Cancer Institute and Department of Pathology, Wayne State University School of Medicine, 110 East Warren Avenue, Detroit, Michigan 48201.Email:dliao@med.wayne.edu mainly used to refer to a tissue and thus has little relevance and application to cell culture where apoptosis is mostly studied (Blagosklonny, 2000). According to the original definition of apoptosis in vivo (Geske et al, 2002; Kerr et al, 1972; Messmer and Pfeilschifter, 2000; Savill and Fadok, 2000), a cell that is dead or in the process of dying, via an apoptotic mechanism, will be phagocytosed by macrophages such that neither antigenic nor toxic components are released to elicit inflammatory response, which often results in scar formation. As distinct from this in vivo process, apoptosis of cultured cells does not involve clearance by macrophages and thus differs from tissue apoptosis at least in the last steps, which are usually mediated by executor caspases. In strong contrast to the voluminous studies with cultured cells, fewer studies have been reported as to whether in various apoptotic tissues where macrophages actively mediate the later steps of the dying process, caspases are still as actively involved as in cultured cells.

If the rate of apoptosis in a tissue exceeds the rate of clearance of the apoptotic cells by macrophages, it is possible that inflammatory and toxic processes could result, leading to a secondary necrosis. This type of situation would be artificially induced by enforced death signaling, such as might occur in a model system driven by a cell death-associated gene (ie, c-myc) or in human tumors treated with chemotherapeutic agents (Aoyagi and Dickson, 1999). These 
situations make it difficult and even impossible to distinguish apoptosis from necrosis in many occasions; thus, programmed cell death, a more general nomenclature, is often used in the description of the cell death. Programmed cell death and apoptosis have been shown to occur via either caspase-dependent or caspase-independent mechanisms (Borner et al, 1999; Chan and Mattson, 1999; Elliott et al, 2000; Hotti et al, 2000; Johnson, 2000; Kolenko et al, 1999; Vier et al, 1999). Although more studies continue to describe the biochemical differences between these two mechanisms, little data have been presented in the literature to morphologically contrast them, especially in tissues. This is important because morphologic features are still the major criteria used to distinguish different types of cell death.

c-Myc oncoproteins are known to play dual roles in cell proliferation and cell death (Conzen et al, 2000; Dang et al, 1999; Hoffman and Liebermann, 1998), similar to many other oncoproteins, such as cyclin D1, E2F1, Ras, and E1A (Chi et al, 1999; Duelli and Lazebnik, 2000; Guo and Hay, 1999). The mechanisms for c-myc-triggered cell death have been addressed extensively in the past decade (Alarcon et al, 1996; Borner and Monney, 1999; Packham and Cleveland, 1995; Prendergast, 1999; Thompson, 1998), but mostly with cultured cells. Both caspase-dependent and caspase-independent pathways have been implicated in the mechanisms for the apoptotic effects of c-Myc in cell culture (Hotti et al, 2000; Juin et al, 1999; McCarthy et al, 1997; Prendergast, 1999; Thompson, 1998). Far fewer studies have addressed the mechanisms of cell death in various c-myc-expressing tissues. In different transgenic mouse models, c-myc has been targeted to the mammary gland (Stewart et al, 1984), liver (Sandgren et al, 1989), lymphocytes (Felsher et al, 1999; Pelengaris et al, 2000), and renal ducts (Trudel et al, 1997). Mammary adenocarcinomas, hepatocarcinomas, lymphomas, and polycystic kidneys develop in these animals, respectively, as the consequences of the expression of the transgene. All of these cancers or benign lesions show many dead cells, which are commonly described as a result of apoptosis. Moreover, the c-myc transgene has also been targeted to the nervous system (Jensen et al, 1998) and the pancreas (Pelengaris et al, 2000); pronounced cell death is also discerned in these transgenic tissues. However, in most of these studies (Christensen et al, 1999; Felsher and Bishop, 1999; Hundley et al, 1997; Pelengaris et al, 2000; Sanders et al, 1999; Sandgren et al, 1989; Stewart et al, 1984; Trudel et al, 1997), including our own (Amundadottir et al, 1996; Liao et al, 2000a; McCormack et al, 1998), the term "apoptosis" has not been applied based on solid morphologic or biochemical evidence; a positive staining of TUNEL is the only indication in most cases. This may be because the concept that c-Myc induces apoptosis has been well established in the past in cell culture systems (Evan et al, 1992; Shi et al, 1992) where it has been well accepted, and this description is simply extended to c-myc-expressing tissue systems in vivo.
In the present study, we described for the first time the detailed morphology of cell death in a tissue expressing a c-myc transgene. We found that mouse mammary tumor cells expressing a c-myc transgene exhibited malformation and degeneration of their mitochondria, in association with a very low level of cytochrome c expression. The dying cells manifested shrinkage and DNA breakage as indicated by a positive TUNEL staining and nuclear localization of apoptosis inducing factor (AIF) but lack of typical apoptotic morphology, including nuclear condensation and formation of cell membrane blebs and apoptotic bodies. The dying process attracted pronounced infiltration of macrophages. The poly(ADP)-ribose polymerase (PARP) was present in the c-myc transgenic tumors mainly as the unprocessed, inactive form. These results suggest that the cell death in the c-myc transgenic mammary tumor tissue may not be a typical apoptosis and may involve a caspaseindependent mechanism.

\section{Results}

\section{Cell Death in Hematoxylin Eosin (HE)-Stained Sections}

We had previously reported that the c-myc transgenic mammary tumors progressed to form specific focal lesions, termed "foci," at the advanced stages. These "tumor-within-a-tumor" foci showed decreased expression of c-myc transgene and different morphology from their surrounding tumor areas (Liao et al, 2000a). The foci could be solid nodules (Fig. 1), glandular structure (Fig. 1A), or a mixture of nodular and glandular types (Fig. 1B). Solid foci rarely contained dead cells, as we had previously reported (Liao et al, 2000a). Glandular foci often contained some dead cells in the cavity of the glands, but the morphology of these dead cells resembled necrosis (Fig. 1D) and therefore is not described in further detail in the present study.

In the nonfocal areas that we termed "the major tumor areas" (Liao and Dickson, 2000; Liao et al, 2000a), there were a large number of dead cells that were usually organized in clusters (Fig. 1, A and C). There always was a space separating a cluster of dead cells from the surrounding viable tumor cells (Fig. 1, A and $\mathrm{C}$ ), which made each cluster of dead cells look like an island in the major tumor areas. Therefore, the clusters of dead cells were termed "apoptotic cell islands" previously (Liao and Dickson, 2000). The reason for calling the dead cells apoptotic cells was primarily because of their positive TUNEL staining (Amundadottir et al, 1996; Liao et al, 2000a). In the present study, the clusters of dead cells are renamed as "dead cell islands" due to their lack of typical morphology of apoptosis as described below.

Formation of dead cell islands seemed to begin with shrinkage simultaneously occurring in several tumor cells (Fig. 1E), which caused a break in cell contact from their neighboring tumor cells. At this stage the cells still showed a morphology similar to their neighboring cells (Fig. 1E). Later in the dying process, nuclear fragmentation with or without chromatin con- 

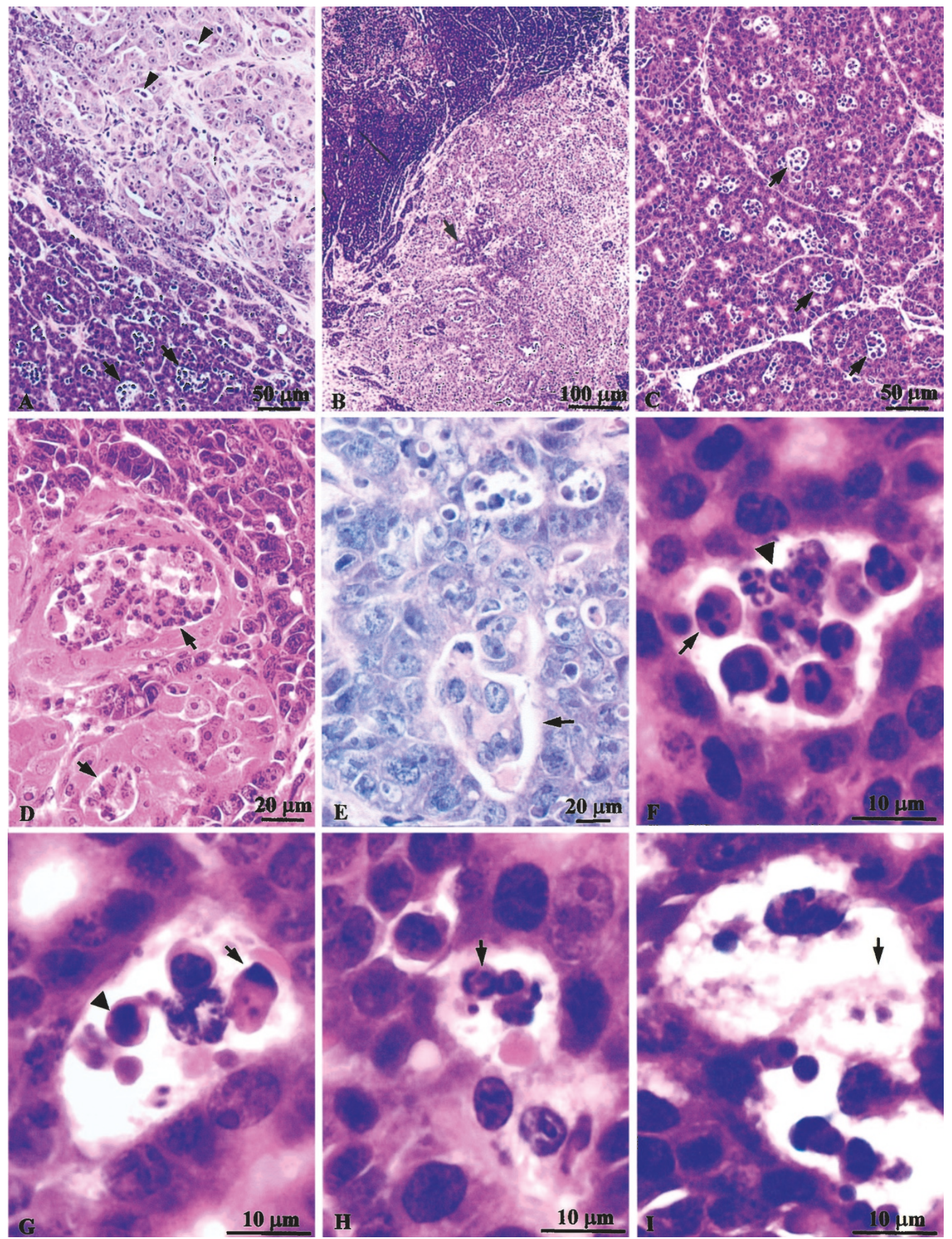

\section{Figure 1.}

Hematoxylin and eosin (HE) staining of c-myc transgenic mammary tumors. Many dead cell islands appear in the major tumor areas (arrowheads in A and arrows in C), but not in the glandular foci (A) nor the mixed type of foci that contains tumor glands (arrow in B). However, there may be some dead tumor cells (arrowheads in $\mathrm{A}$ and arrows in D) in the tumor glands that resemble necrosis. At early stage of dead cell island formation, a group of tumor cells (arrow in E) are separate from their surrounding tumor cells by an intercellular space that might have resulted from cell shrinkage; at this time point, the morphology of these cells is still similar to the surrounding cells. Cells in dead cell islands show nuclear fragmentation (arrow in F and G), nuclear condensation (arrowhead in G) or peripheral chromatin condensation (arrow in $\mathrm{H}$ ). There are many small nuclear fragment-like components inside one giant cell (arrowhead in F), which may be a macrophage. Cell debris appears in a dead cell island (arrow in I). 
densation was discerned as the main alteration of the cells (Fig. 1, F and G). Peripheral chromatin condensation could also be observed (Fig. $1 \mathrm{H})$. Disruption of cell membranes and cytoplasm seemed to be common, leaving much cell debris in the hollow dead cell islands (Fig. 1l). Giant cells containing many small, fragmented, and nucleus-like components were frequently observed (Fig. 1F). Considering the results of toluidine blue staining (see below), these cells might be macrophages with phagosomes containing nuclear fragments, although their true identity could not be ascertained with HE staining. Blebs of cell membrane and apoptotic bodies resulting from cell membrane blebbing were never observed, even when carefully examined under high magnification.

Hemorrhage was discerned in some of the dead cell islands. However, an obvious inflammatory response, characterized by infiltration of many white blood cells, was never observed in dead cell islands.

\section{Cell Death in Toluidine Blue-Stained Sections}

Small tumor tissue blocks were fixed with glutaraldehyde and osmium tetroxide, stained with uranyl acetate, and then embedded with Spurr resin. Semi-thin sections $(1 \mu \mathrm{m})$ were made and stained with toluidine blue. Therefore, the staining observed might result from not only toluidine blue but also osmium tetroxide and uranyl acetate. The staining identified two major types of tumor cells: dark cells and light cells (Fig. 2, A and $B$ ). The two types of cells were roughly equal in number. Either type could dominate an area or they could be variously mixed with each other (Fig. 2, A and B).

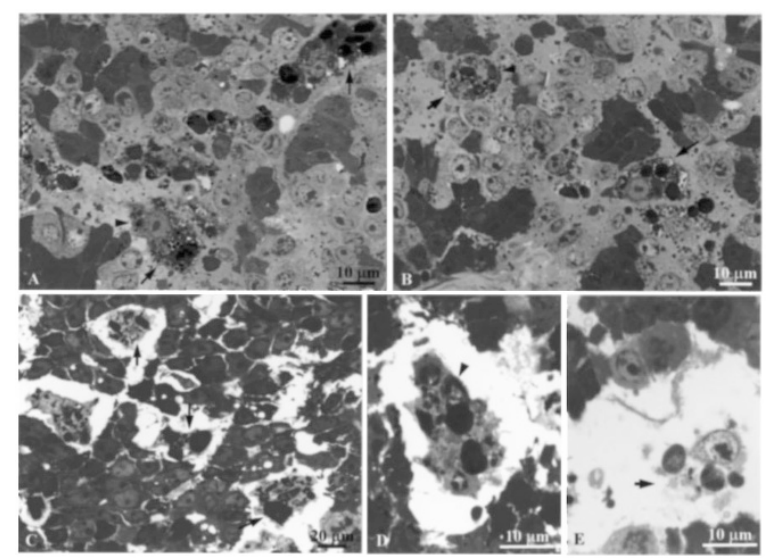

Figure 2.

Toluidine blue staining of c-myc transgenic mammary tumor tissue that was prestained with osmium tetroxide and uranyl acetate. Two types of tumor cells light and dark cells are identified $(A$ and $B)$. Note that few dead tumor cells per se appear in the dead cell islands ( $A$ and $B$ ), except several huge macrophages (arrow) containing many intensely stained phagosomes. Macrophages could also appear among the viable tumor cells (C). Some phagosomes show a staining density as light as the cytoplasm of viable tumor cells, while some other phagosomes contain intensely stained components (arrowhead in A, B, and D). These light-stained phagosomes could contain entire engulfed dead tumor cells, while the dark components within them might be nuclear fragments. A disrupted cell is indicated in E; its large body size and phagosomes suggest that it may be a dead macrophage.
A large number of macrophages was observed in the tumor tissue, more frequently colocalized with dead cell islands (Fig. 2, A and B) but sometimes also among viable tumor cells (Fig. $2 \mathrm{C}$ ) or in the thin stroma. The macrophages were very large in size, usually about 5- to 8-fold larger than viable tumor cells. Without exception all macrophages contained many phagosomes that varied greatly in size; the phagosomes were frequently as large as the nucleus of viable tumor cells (Fig. 2D). Many phagosomes were intensely stained, while in others only some components showed intense staining (Fig. 2, A to D). These intensely stained phagosomes or the phagosomal components were likely to be the nuclei or nuclear fragments of dead cells engulfed in macrophages. In conflict with the observations from HE-stained sections, few dead tumor cell nuclei were found in dead cell islands, and nuclear condensation or peripheral chromatin condensation was not observed in dying or dead tumor cells. Thus, many dead cell nuclei with or without nuclear condensation observed in the HEstained sections might not be the nuclei of dead cells per se, but might actually be the nuclei or nuclear fragments of the phagocytosed tumor cells appearing as phagosomes or phagosomal components within macrophages.

Consistent with what was observed in HE-stained sections, blebs of cell membrane were not observed in the tumor cells, although cellular structures were preserved better with glutaradehyde fixation, and membrane blebbing should be discerned more easily. Disruption of tumor cells was rarely seen, which was in conflict with the observation of cell debris in the dead cell islands in HE-stained sections. On the other hand, disrupted macrophages were often observed, releasing phagosomes as well as other cellular components to the intercellular space as cell debris (Fig. 2E). It is likely that the disrupted cells and cell debris observed in HE-stained sections might actually be disrupted macrophages.

\section{TUNEL Staining}

Many, but not all, nuclei in dead cell islands were positive for TUNEL staining (Fig. 3, A, C, and D), indicating that DNA breakage occurred in these cells, because the principle for TUNEL staining is DNA end-labeling. Interestingly, in the hyperplastic lesions of mammary epithelia, TUNEL-positive nuclei appeared individually but were not organized in clusters (Fig. 3B). This difference implies that although cell death in both hyperplastic lesions and tumors might all be triggered by enforced expression of c-myc, the mechanisms for the cell death might still be distinct, comparing the benign lesions with the tumors.

In conflict with the observation of HE-stained sections, most TUNEL-positive nuclei in dead cell islands did not show condensed chromatin under high magnification; instead, their density was very similar to that of the nuclei of viable tumor cells, except for the brown TUNEL staining (Fig. 3, A, C, and D). Moreover, in dead cell islands, many nuclei positive for TUNEL 

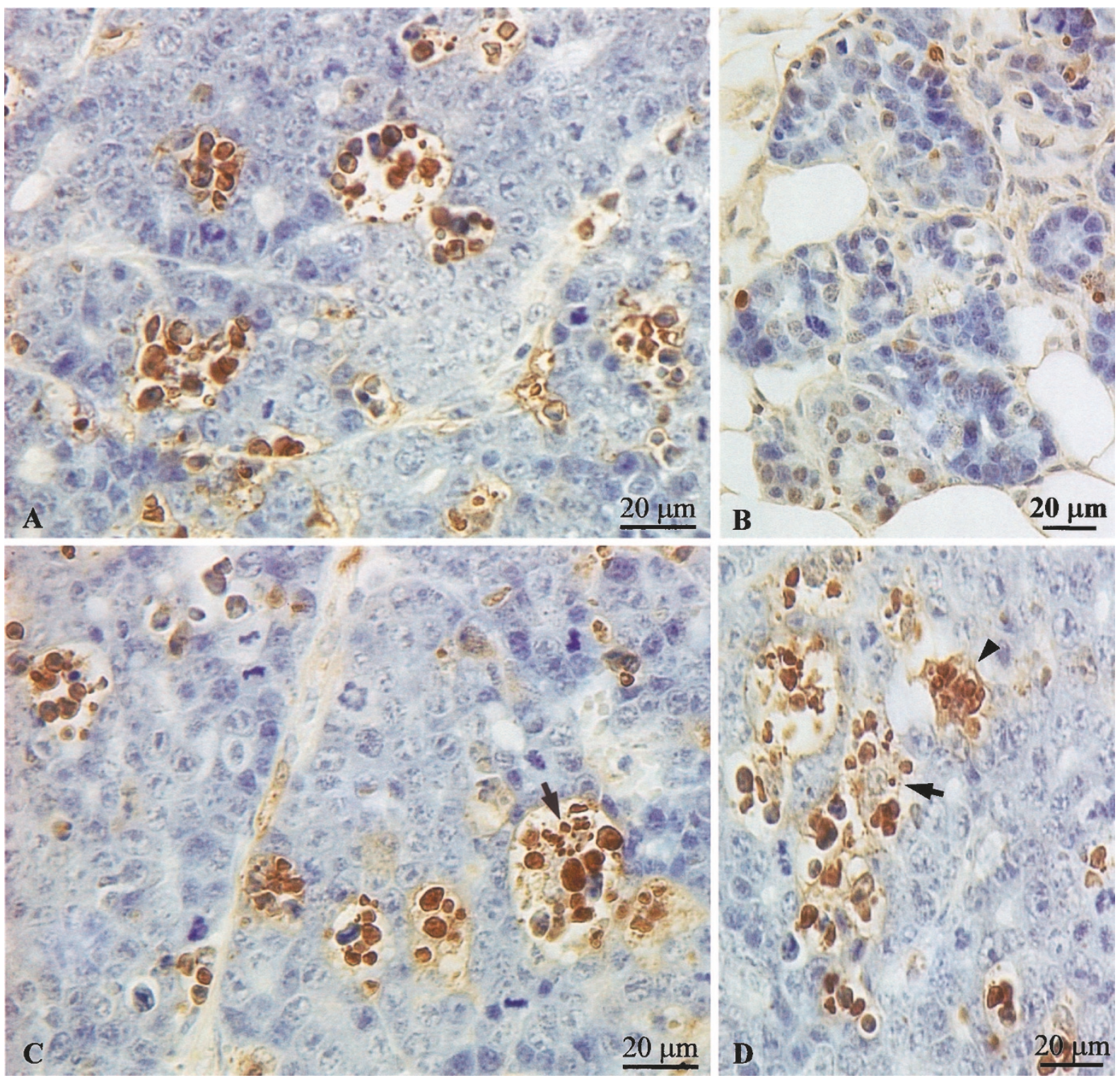

Figure 3.

TUNEL staining (brown color) of c-myc transgenic tumor tissue (A, C, and D) and hyperplastic lesion (B), with hematoxylin-counter staining (blue color). Positive nuclei in the tumor tissue appear in clusters to form dead cell islands (A, C, and D), whereas positive nuclei in a hyperplastic lesion adjacent to a tumor area appear individually, without formation of dead cell islands. The chromatin density of the positive nuclei in dead cell islands is similar to that of the viable tumor cells. There is no cytoplasm surrounding most of the positive nuclei within dead cell islands, although the cytoplasm of the viable tumor cells can be discerned clearly by its weak hematoxylin staining. There are some small positive particles embedded in cytoplasm (arrow in C and D), which could be nuclear fragments inside macrophages Several positive nuclei are encapsulated by cell membrane (arrowhead in D), indicating that they might be phagocytosed by a macrophage.

were surrounded by a hollow space but not by a cytoplasm and cell membrane, although the cytoplasm of the viable tumor cells was clearly discerned by its weak hematoxylin staining. This result indicates that the cytoplasm and cell membrane of these dead cells might have already lysed and dispersed, which might explain why no cell membrane blebs and apoptotic bodies were observed. The lysis might occur after these tumor cells are engulfed by macrophages, because disruption of tumor cells per se was rarely seen in toluidine blue-stained sections. This consideration was supported by the observation that many nucleus-like, but smaller than a nucleus, particles were also positive for TUNEL (arrow in Fig. 3, C and D).
Frequently, these small positive particles were embedded in a cytoplasmic-like component (arrow in Fig. $3, C$ and D), indicating that they were nuclear fragments of a dead tumor cell engulfed and digested by macrophages. More convincing support for this conclusion was the common observation that a group of positive nuclei were encapsulated by a membrane (arrowhead in Fig. 3D).

\section{Electron Microscopic (EM) Observations}

There were also two main types of tumor cells identified under EM: dark cells and light cells (Fig. 4A), corresponding to the dark and light cells in toluidine 


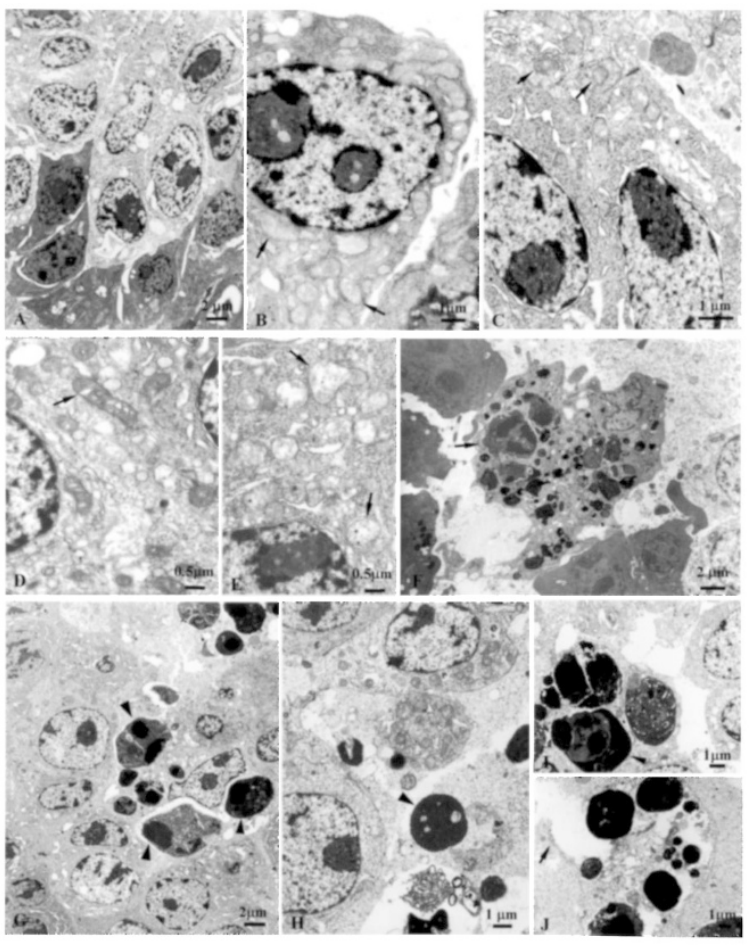

Figure 4.

Ultrastructure of c-myc transgenic tumor tissue. According to their electron density, the tumor cells could be divided into two categories of dark cells and light cells $(A)$. Most tumor cells show one or more giant nucleoli ( $A$ and $B$ ). Mitochondria (arrow) of the tumor cells show either electron-light ( $B$ and $C$ ) or electron-dark (D) amorphous matrix, with absent or very few cristae. When the cristae appear, they may be dilated to cavities with irregular shapes (arrow in D). Frequently, the malformed mitochondria are degenerated, characterized by lysis of matrix and cristate, leaving a hollow mitochondrial body (arrow in E). Macrophages appear frequently, especially in the places of dead cell islands ( $F$ and $\mathrm{G}$ ). Every macrophage contains many phagosomes (arrowhead in $\mathrm{F}$ to $\mathrm{J}$ ). Some phagosomes show very high electron density ( $H$ and $J)$ or contain components with very high electron density (I). Many phagosomes could be as large as a nucleus $(G)$, and some resemble the nucleus of engulfed cell (arrow in $F$ ). Note that in the place of dead cell islands $(G)$, no tumor cells show condensed nucleus. Disruption of cell membrane and release of phagosomes is discerned in a macrophage (arrow in $\mathrm{J}$ ).

blue-stained sections. The nuclei of both types of tumor cells distinctively showed one or more giant nucleoli. Most dark tumor cells showed an undifferentiated and inactive ultrastructure, characterized by electron-dense, amorphous matrix in the cytoplasm, with very few organelles such as endoplasmic reticulum and Golgi apparatus. The light tumor cells usually contained more organelles compared with the dark tumor cells. Lysosomes, glycogen granules, and lipid droplets were easily discerned in the light tumor cells (in contrast to dark cells), in addition to larger numbers of endoplasmic reticulum and Golgi complexes. Autophagosomes were also discerned in some light cells.

For both cell types, the most striking alterations were in the mitochondria. In most tumor cells, the mitochondria consisted mainly of an electron-light (Fig. 4, B and C) or electron-dense (Fig. 4D) amorphous matrix, with very few cristae (Fig. 4, B to D). The cristae were usually dilated to the cavities with irregular shapes (arrow in Fig. 4D). These morphologic features suggest that the mitochondria are malformed in these c-myc-expressing tumor cells. Moreover, these malformed mitochondria were frequently swollen and degenerated, characterized by the lysis of the amorphous matrix and cristae, leaving a hollow mitochondrial body (Fig. 4E).

EM examination also confirmed the presence of many macrophages in the tumor tissue, especially in dead cell islands (Fig. 4, F and G). Most macrophages were so huge and so irregular in their shapes that it was usually impossible to take a photo of the whole cell, even under the lowest magnification. All macrophages contained many phagosomes, with various degrees of digested tumor cells, indicating that the macrophages were very active in phagocytosis. The electron density of the phagosomes varied greatly, and it was common that one phagosome showed very high electron density in one part but very light density in the other part (Fig. 4, G, I, and J). The light and dark parts resulted from different organelles and nuclei that were phagocytosed, and there usually was a clear edge between the light and dark parts (Fig. 4, G and I). Peripheral chromatin condensation and nuclear condensation were not seen in dying or dead tumor cells, which was in contrast to the observation of HEstained sections but dovetailed with the observation of the sections stained for toluidine blue and TUNEL. However, when the electron-dense components of a phagosome resided in the peripheral area or in a corner, it might make the phagosome resemble peripheral chromatin condensation under light microscope. Thus, the structures that resembled condensed nuclei or peripherally condensed chromatin observed in HE-stained sections were unlikely to be the nuclei of dead tumor cells per se but might be the phagosomes containing residuals of nuclei.

Disruption of macrophages with release of cell debris and processed phagosomes to the intercellular space was very commonly observed (Fig. 4, H to J). On the other hand, formation of cell membrane blebs and apoptotic bodies in dying or dead tumor cells were not observed. Disruption of dead tumor cells was rarely seen. These results again suggest that the cell debris in dead cell islands observed in HE-stained sections might be derived mainly from disrupted macrophages.

\section{Expression of Cytochrome c, AIF, and CD68}

Because the mitochondria in the tumor cells were found to be malformed and frequently degenerated, we performed immunohistochemical staining of cytochrome $\mathrm{c}$ and AIF, two mitochondrial proteins known to mediate caspase-dependent and caspaseindependent apoptosis, respectively (Daugas et al, 2000a, 2000b; Loeffler and Kroemer, 2000; Lorenzo et al, 1999; Skulachev, 1998), although AIF is also related to necrosis (Daugas et al, 2000b). Most tumor cells in the major tumor areas and some cells in the dead cell islands exhibited positive staining of cytochrome $c$ in the cytoplasm (Fig. 5, A to D). However, to our surprise, the staining intensity was much weaker compared with the staining in the tumor foci or in the 



\section{Figure 5.}

Immunohistochemical staining of cytochrome c, apoptosis inducing factor (AIF), and CD68 in c-myc transgenic mammary tumor tissue. Staining of cytochrome c is positive in major tumor areas and in some (but not all) dead cell islands (arrow in C), but the staining intensity is much weaker compared with the smooth muscle cells (upper part in $\mathrm{A}$ ) and the adjacent foci (B to D). Under high magnification, the staining of cytochrome $\mathrm{c}$ in both foci and surrounding tumor area is granular in the cytoplasm (D). AIF staining is localized to some nuclei in the dead cell islands (E and F). Staining for CD68, a marker of macrophages, identifies the positive cells in dead cell islands $(\mathrm{G})$, in stroma, and among the tumor cells $(\mathrm{H})$. The staining is localized to the cytoplasm and probably also cell membrane, in contrast to the nuclear staining of AlF. 
smooth muscle cells that were used as internal positive controls (Fig. 5, A and B). This result suggests that a higher expression of cytochrome c can be used as an additional marker for the tumor foci. Under high magnification the staining was found to be granular in the cytoplasm (Fig. 5, C and D), indicating that the cytochrome c might still be localized in the mitochondria. Western blot assay of total c-myc tumor tissue revealed a single band at the expected molecular weight (Fig. 6), confirming the specificity of the antibody. A normal rabbit Ig $G$ was used to replace the primary antibody in some control staining; this control did not result in any signal (not shown).

Immunohistochemical staining of AIF showed that some, but not all, cells in the dead cell islands were positive in their nuclei, while cells in the major tumor areas were negative or very weakly positive in the cytoplasm (Fig. 5, E and F). Cells in the foci were negative. Western blot analysis of the total tumor tissue showed a single band at the expected molecular weight (Fig. 6), confirming that AIF was expressed in the tumor tissue.

Immunohistochemical staining of macrophage marker CD68 gave rise to positive staining in the cytoplasm of many cells. The positive cells were found mainly in the dead cell islands (Fig. 5, G and H), usually around the dead cell nuclei, indicating that these nuclei might actually be phagocytosed inside the giant macrophages. The positive cells were also found in the thin stroma among the viable tumor cells (Fig. $5 \mathrm{H}$ ) but were very rare in the foci (not shown). Western blot assay with total tumor tissue lysates showed a single band at the expected molecular weight (Fig. 6), suggesting the specificity of the antibody. Because the CD68 and AIF antibodies were of goat origin, they functioned as each other's control for the staining specificity. Nevertheless, a normal goat Ig G was also used to replace the primary antibodies for some control staining, which did not result in any signal,

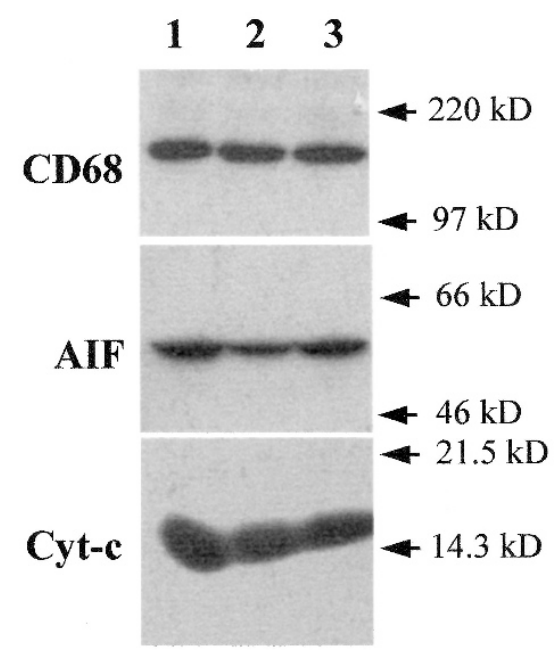

Figure 6.

Western blot analysis detects cytochrome c, apoptosis inducing factor, and CD68 proteins in total tissue lysates prepared from three (Lanes 1 to 3 ) randomly selected c-myc transgenic mammary tumors. confirming the staining specificity yielded by the CD68 and AIF antibodies.

\section{Expression of Caspase-3 and PARP}

Caspase-3 was analyzed in the total tissue lysates of 10 MMTV-c-myc transgenic mammary tumors using Western blot assay. Five MT-tgf $\alpha$ MMTV-c-myc double transgenic tumors were included as negative controls because these tumors were basically nonapoptotic (Amundadottir et al, 1996; Liao et al, 2000a). The protein levels of caspase-3 varied greatly among different c-myc tumors but in general were very low and similar with the levels in the tgf $\alpha$ /c-myc double transgenic tumors in which dead cells were rarely seen (Amundadottir et al, 1996; Liao et al, 2000a). Figure 7 shows the results of three c-myc tumors and two $\operatorname{tgf} \alpha / \mathrm{c}-m y c$ bigenic tumors as the representatives. The caspase- 3 was detected mainly as the unprocessed protein at $32 \mathrm{kD}$, whereas the cleaved, active form at $17 \mathrm{kD}$ was detectable only in 1 of the $10 \mathrm{c}-$ myc tumors (Lane 3 in Fig. 7) and 1 of the 5 (Lane 4 in Fig. 7) bigenic tumors, suggesting that caspase- 3 might not be involved in the c-Myc-initiated cell death. To confirm that the primary antibody works at a high sensitivity for the mouse caspase-3, a mammary tumor cell line, Myc83, which was originally established from an MMTV-c-myc transgenic mouse by our laboratory, was treated with anisomycin (100 $\mu \mathrm{m}$ for 24 hours) in culture to induce caspase-dependent apoptosis. Caspase-3 in these apoptotic Myc83 cells was expressed abundantly and was present as both the unprocessed and the processed forms (Fig. 7). Immunohistochemistry with the same caspase-3 antibody showed a very weak cytoplasmic staining in the viable tumor cells of the major tumor areas but not in the dead cell islands (data not shown).

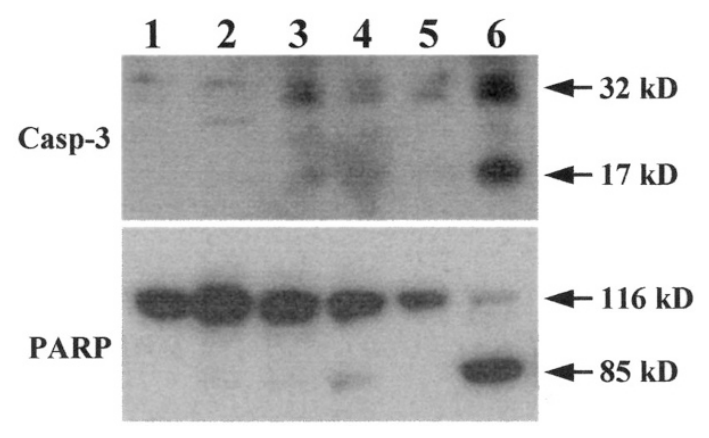

Figure 7.

Western blot analyses of caspase-3 (Casp-3) and poly(ADP)-ribose polymerase (PARP). Lanes 1 to 3 (150 $\mu \mathrm{g}$ proteins per lane) are three representative MMTV-c-myc transgenic mammary tumors, whereas Lanes 4 and 5 (150 $\mu \mathrm{g}$ proteins per lane) are two representative MT-tgf $\alpha /$ NNTV-c-myc double transgenic mammary tumors. Lane 6 (20 $\mu$ g protein) is Myc 83 mouse mammary tumor cells induced to undergo apoptosis by the treatment with anisomycin. The levels of caspase-3 varied largely among either c-myc or tgf $\alpha / \mathrm{c}-m y c$ tumors but were in general very low. It was mainly present as the unprocessed form at $32 \mathrm{kD}$. In contrast, caspase-3 in the apoptotic Myc83 cells was abundantly expressed and was present as both the unprocessed form at $32 \mathrm{kD}$ and the cleaved form at $17 \mathrm{kD}$. PARP was expressed at high levels and was present mainly as the unprocessed form at $116 \mathrm{kD}$ in all tumors but was present mainly as the cleaved form at $85 \mathrm{kD}$ in the apoptotic Myc83 cells. 
On Western blot, PARP was detected mainly as the unprocessed protein at $116 \mathrm{kD}$ in both c-myc and $\operatorname{tgf} \alpha / \mathrm{c}-m y c$ tumors but was present mainly as the cleaved form at $85 \mathrm{kD}$ in the apoptotic Myc83 cells (Fig. 7). Immunohistochemical staining for PARP was mainly localized in the nuclei of the viable tumor cells in the major tumor areas but not in the dead cell islands (data not shown). The two PARP primary antibodies $(\mathrm{H}-250$ and $\mathrm{SA}-252)$ used gave rise to the same Western blot and immunohistochemical results.

\section{Discussion}

Mammary gland tumors spontaneously developed in the MMTV-c-myc transgenic mice are known to contain many dead and dying cells (Liao and Dickson, 2000); the rate of these cells in the nonfocal areas (ie, the major tumor areas) is about $16 \%$, as quantified by positive TUNEL staining in our previous report (Liao et al, 2000a). The dying and dead tumor cells were uniquely organized in clusters, which were termed by us as dead cell islands. One fundamental question on our observations is whether the formation of dead cell islands is a result of the c-myc overexpression. Although we have no direct evidence to tightly connect c-myc overexpression with the formation of dead cell islands, due to limitations in our model system, there are several indications suggesting this connection. First, the dead cell islands are observed only in the major tumor areas that show high levels of c-myc but not in the foci that have lost the c-myc expression (Liao et al, 2000a). Second, similar clusters of dead cells have also been reported in the polycystic kidney of mice where a c-myc transgene was targeted to the renal ducts (Trudel et al, 1997). Third, to our knowledge a similar morphology of dead cell islands has not been described in the literature for cell death triggered by any other factors in any other types of tissue, although this could be due to the lack of detailed morphologic descriptions in most tissue-based studies. One of the possible explanations for the appearance of dead cell islands is that cells in a local neighborhood may commit collective suicide, which indirectly implies the involvement of a paracrine mechanism or a cell-cell interaction in the dying process.

Although cell death in c-myc-overexpressing tissues has previously been referred to by us as apoptosis (Amundadottir et al, 1996; Liao et al, 2000a; McCormack et al, 1998) and other investigators (Christensen et al, 1999; Hundley et al, 1997; Pelengaris et al, 2000; Sanders and Thorgeirsson, 1999; Sandgren et al, 1989; Stewart et al, 1984; Trudel et al, 1997), actually no morphologic or biochemical evidence other than a positive TUNEL staining has been provided to prove that the cells really died from an apoptotic mechanism in any of these tissues. In the present study, we found that cell death in the c-myc transgenic mammary tumors lacks various morphology of apoptosis, including chromatin condensation, cell membrane blebbing, and apoptotic body formation. However, the dying process involves many macrophages, which is a typical phenomenon of in vivo apoptosis. As a consequence, it is the phagosomes that are released to the intercellular space but not the apoptotic bodies. The released phagosomes have been processed and membrane-capsulated and thus do not elicit inflammatory response. These in vivo morphologic features do not seem to fit into the typical description of apoptosis, necrosis, or any other types of cell death provided by the literature, although they are probably closer to "delayed necrosis" or "slow cell death" as termed by Blagosklonny to describe the cell death without caspase activation (2000). Currently it is unclear if the morphologic features observed by us are specific for the c-myc transgenic mammary tumors or if it is common for any of the c-myc-expressing tissues. Because many morphologic properties of apoptosis are described mainly based on observation of cultured cells without involvement of macrophages, more thorough studies are needed to explore whether certain morphologic features of apoptosis are really common phenomena in various apoptotic tissues where many macrophages are involved. Examples of such apoptotic features include nuclear condensation, cell membrane blebbing, and apoptotic body formation. It cannot be excluded that the latest steps of programmed cell death are catalyzed by some enzymes of macrophages but not by caspases of the dying cells.

Malformation of mitochondria and depletion of cytochrome c have been reported in the cultured 3T3 cells ectopically expressing $\mathrm{Bcl}-\mathrm{Xs}$, an apoptosisinducing protein (Fridman et al, 2001). In these cells mitochondria appear less electron-dense or even transparent. The cristae of mitochondria in these cells are either undeveloped or broken. Similar malformation and degeneration of mitochondria are observed in the c-myc-expressing mammary tumor cells in the present study, which shows for the first time in a tissue that enforced expression of an inducer of programmed cell death may cause malformation and degeneration of mitochondria. It is possible that the pro-death property of these c-myc-expressing cells may be causally related to these mitochondrial alterations.

Expression of cytochrome $\mathrm{c}$ is much lower in dead cell islands and in most c-myc-expressing tumor cells compared with the c-myc-silenced foci and to the adjacent muscle cells that require high levels of cytochrome c for energy generation. These surprising findings are not a general phenomenon occurring to all mitochondrial proteins because AIF does not change similarly. These alterations may be explained by an inhibition of cytochrome $\mathrm{c}$ in the majority of the tumor cells in a way similar to the possible suppression of cyclin D1 by c-Myc shown previously (Liao et al, 2000a). Alternatively, it may also be explained by an induction of cytochrome $c$ in the foci due to a higher demand of energy generation for a higher proliferating rate of the foci (Liao et al, 2000a). It has been known that $c-M y c$ induces lactate dehydrogenase gene $(L D H-A)$ in fibroblasts and that c-myc-expressing cells are enhanced for glycolytic energy generation, a pathway with a much lower efficacy (Osthus et al, 
2000; Shim et al, 1997, 1998a). Glucose depletion has been shown to induce apoptosis of c-myc-expressing Rat1 cells in culture (Shim et al, 1998b). The aforementioned $\mathrm{Bcl}-\mathrm{Xs}$-expressing cells that show mitochondrial malformation and cytochrome c depletion undergo caspase-independent cell death (Fridman et al, 1999, 2001). Putting these data together, one may hypothesize that insufficient generation of energy, due to the reasons like damage in mitochondria or depletion in cytochrome c or glucose, may be capable of inducing programmed cell death, which may not be a typical apoptosis because apoptosis is defined as a process that involves consumption of energy (Blagosklonny, 2000). In support of this hypothesis, cultured fibroblasts isolated from cyctochrome c knockout mouse, which are devoid of cytochrome $c$ and show reduced caspase- 3 activation, also exhibit an increased sensitivity to TNF $\alpha$-induced apoptosis, compared with the wild-type fibroblasts (Li et al, 2000; Vier et al, 1999). It is possible that c-myc transgenic tumor cells die from this energy-insufficient mechanism because they exhibit damage of mitochondria, low level of cytochrome c, and lack of several morphologic properties of apoptosis.

In a reported study (McCarthy et al, 1997), when Rat1/c-MycER cells were induced by c-Myc expression to undergo apoptosis in the absence of caspase inhibitors, they showed typical apoptotic morphology such as chromatin condensation, cell shrinkage, cytoplasmic blebbing, and cell fragmentation. In contrast when the cells were induced to undergo apoptosis in the presence of caspase inhibitors, although the cells still showed cytoplasmic blebbing, their nuclei remarkably resembled those of nonapoptotic cells. These results indicate that c-myc-triggered cell death may have both caspase-dependent and caspaseindependent mechanisms (Daugas et al, 2000a, 2000b); in the latter situation, the nuclear morphology was similar to what was observed in our study. The lack of PARP cleavage suggests that programmed cell death in the c-myc tumors may be caspaseindependent because PARP cleavage is a common downstream event of all known caspase-dependent pathways. Other data supporting this consideration include the low level of cytochrome $c$ in dead cells, the nuclear localization of AIF in dead cells, and the low level of caspase-3. The lack of nuclear apoptotic features such as chromatin condensation also dovetails with this consideration, because it now seems to be clear from the literature that inhibition of caspases can prevent nuclear apoptotic changes (Hirsch et al, 1997; Kolenko et al, 1999). Thus, the morphologic features observed in the present study might just be a reflection of the differences between caspasemediated apoptosis and caspase-independent, nonapoptotic programmed cell death, which is an issue with a significant amount of very confusing data from different cultured cells but with too little data from tissues. More thorough studies need to be conducted on the relationship between the morphologic features of these two dying processes and the status of caspase activation in various apoptotic tissues.
In summary c-myc-expressing mammary tumor cells exhibited malformation and degeneration of mitochondria, with a much lower protein level of cytochrome $\mathrm{c}$ than in the c-myc-silenced foci, for which a higher level of cytochrome $\mathrm{c}$ is an additional marker. The dying procedure involved cell shrinkage, DNA breakage, and nuclear localization of AIF, but without nuclear condensation and formation of cell membrane blebs and apoptotic bodies. In addition, this process of cell death involved a pronounced infiltration of macrophages. PARP was present mainly as the unprocessed, inactive form. Collectively, these results suggest that the programmed cell death of the c-myc transgenic mammary tumor cells may not be a typical apoptosis and may be caspase-independent. It deserves further study as to whether the cell death process, appearing in other c-myc-expressing tissues, is also distinct from classical apoptosis.

\section{Materials and Methods}

\section{Tissue Collections}

Virgin female MMTV-c-myc transgenic mice described before (Amundadottir et al, 1996; Liao et al, 2000a) were housed until spontaneous mammary tumors developed. When the tumors reach about 15 to $20 \mathrm{~mm}$ in diameter, usually at the age of 9 to 12 months, the animals were killed for tumor collection. Part of the tumor tissue was sliced into thin pieces and immediately fixed with $10 \%$ formalin in PBS $(\mathrm{pH} 7.4)$ overnight at $4^{\circ} \mathrm{C}$. The tissue was subjected to a quick (6 hours) procedure of tissue processing and was embedded with paraffin. In total, 30 paraffin-embedded tumors were sectioned $(5 \mu \mathrm{m})$ and $\mathrm{HE}$ stained. Other parts of tumor tissues were first frozen in liquid nitrogen and stored at $-80^{\circ} \mathrm{C}$ for further molecular analyses of the tumors. In addition, five mammary tumors from virgin female MT-tgf $\alpha$ /MMTV-c-myc double transgenic mice collected from previous experiments (Amundadottir et al, 1996; Liao et al, 2000a) were also used as controls for the expression of caspase- 3 and PARP.

\section{Toluidine Blue Staining and EM}

After killing the animals, six tumors were prepared for EM. For this purpose, fresh tissue biopsies collected from five different places in each tumor were chopped into about $1-\mathrm{mm}^{3}$ pieces and then immediately fixed with $3 \%$ glutaraldehyde in PBS $(\mathrm{pH} 7.2)$ overnight at $4^{\circ} \mathrm{C}$. The glutaraldehyde-fixed tumor tissues were postfixed with $1 \%$ osmium tetroxide (Sigma Chemical Company, St. Louis, Missouri) for 1 hour and washed with distilled water three times (10 minutes each). The tissues were stained with $2 \%$ uranyl acetate (Sigma Chemical Company) for 30 minutes in the dark and washed with distilled water three times, followed by dehydration with graded ethanols and then propylene oxide. The tissues were incubated in a mixture of propylene oxide and Spurr resin overnight and then in Spurr for 6 hours. The tissues were embedded in Spurr and cured in an oven at $65^{\circ} \mathrm{C}$ overnight. Semithin sections $(1 \mu \mathrm{m})$ were cut with Reichert Ultracut 
microtome and stained with $1 \%$ toluidine blue in $1 \%$ borax. Ultrathin sections $(70 \mathrm{~nm})$ were cut with the same microtome, mounted on 200 mesh grids, and stained with lead citrate. The sections were viewed on JEOL 1200EX electron transmission microscope.

\section{TUNEL Assay}

The terminal deoxynucleotidyl transferase (TdT) mediated digoxigenin-dUTP nick end labeling (TUNEL) method was carried out using a kit from Trevigen Inc. (Gaithersburg, Maryland) as described previously (Liao et al, 2000a). Paraffin sections (5 $\mu \mathrm{m})$ were dewaxed, rehydrated, treated with protease $\mathrm{K}$, and blocked with $\mathrm{H}_{2} \mathrm{O}_{2}$, according to the manufacturer's instructions. After labeling with TdT and biotin-labeled dNTP, the sections were incubated with peroxidaseconjugated streptavidin. The signal was visualized by exposure to diaminobenzidine and $\mathrm{H}_{2} \mathrm{O}_{2}$, followed by counter-staining with hematoxylin.

\section{Immunohistochemistry}

Immunohistochemical staining was performed using an avidin-biotin complex method described previously (Liao et al, 2000a). Paraffin sections were deparaffinized and endogenous proxidase activity was blocked with 3\% peroxide. Antigens were retrieved by heating in a microwave oven in 50-mm citrate buffer, $\mathrm{pH}$ 6.0, after boiling for 8 minutes. After blocking nonspecific antigens with $6 \%$ normal goat or horse serum, the sections were incubated with the primary antibody for 2 hours, followed by 1 hour incubation with a second antibody conjugated with biotin (Vector Laboratories Inc., Burlingame, California). The sections were then incubated with peroxidaseconjugated avidin (Dako, Corporation, Carpinteria, California) for 30 minutes, followed by color development with diaminobenzidine and peroxide. All procedures were carried out at room temperature. The primary antibodies used were rabbit polyclonal cytochrome c antibody ( $\mathrm{H}-104)$, goat polyclonal CD68 antibody (M-20), goat polyclonal antibody (D-20) against AIF, and rabbit polyclonal antibody $(\mathrm{H}-250)$ against PARP, all from Santa Cruz Biotechnology Inc., Santa Cruz, California. Other primary antibodies used were rabbit polyclonal antibody (M042453) against caspase-3 from Pharmingen and rabbit polyclonal antibody against PARP (SA-252) from Biomol Research Labs Inc., Plymouth Meeting, Pennsylvania. To control for signal specificity, serial sections were made from four tumors and were subjected to the same staining procedure, with a normal rabbit or goat lg $\mathrm{G}$ to replace the respective primary antibody. This control staining did not give rise to a signal, demonstrating the specificity of the signal given by the primary antibodies.

\section{Western Blot Analysis}

Frozen tumor tissues were homogenized with a polytron in a lysate buffer containing (Liao et al, 2000a, 2000b): 50 mm Tris- $\mathrm{HCl}$ (pH 7.4), $150 \mathrm{~mm} \mathrm{NaCl,} 2 \mathrm{~mm}$
EDTA, 0.5\% NP-40, $50 \mathrm{~mm} \mathrm{NaF,} 0.5 \mathrm{~mm} \mathrm{Na}_{3} \mathrm{VO}_{4}, 20$ mM sodium pyrophosphate, $1 \mathrm{~mm}$ PMSF, $10 \mu \mathrm{g} / \mathrm{ml}$ aprotinin, $10 \mu \mathrm{g} / \mathrm{ml}$ leupeptin, and $1 \mathrm{~mm}$ DTT. The tissue lysates were transferred to a B-type glass homogenizer, homogenized again, and centrifuged at $12,000 \mathrm{rpm}$ for 20 minutes at $4^{\circ} \mathrm{C}$. The supernatants were collected, and their protein concentrations measured with BCA reagents (Pierce, Rockford, Illinois). Protein aliquots were electro-fractionated on SDSPAGE. Roughly equal loading was confirmed by staining the gel with Coomassie blue, although comparisons among different samples were not emphasized. The proteins were transferred onto a PVDF membrane and probed with primary antibody for 2 hours, followed by incubation with a peroxidase-conjugated second antibody for 1.5 hours. The signal was visualized with chemiluminescent substrates (Pierce) and autography. The primary antibodies used in Western blot analyses were the same as used for immunohistochemical staining. For each target gene, Western blot was repeated three times, and a representative figure was presented.

\section{Acknowledgements}

We would like to express our gratitude to M-Z Dai for help with electron microscopy and Dr. Gloria Chepko for helpful discussions and other assistance with this study.

\section{References}

Afford S and Randhawa S (2000). Apoptosis. Mol Pathol 53:55-63.

Alarcon RM, Rupnow BA, Graeber TG, Knox SJ, and Giaccia AJ (1996). Modulation of c-Myc activity and apoptosis in vivo. Cancer Res 56:4315-4319.

Amundadottir LT, Nass SJ, Berchem GJ, Johnson MD, and Dickson RB (1996). Cooperation of TGF alpha and c-Myc in mouse mammary tumorigenesis: Coordinated stimulation of growth and suppression of apoptosis. Oncogene 13:757765.

Aoyagi $\mathrm{H}$ and Dickson RB (1999). Programmed cell death and its resistance in breast cancer chemotherapy. Pathogenesis 13:143-162.

Blagosklonny MV (2000). Cell death beyond apoptosis. Leukemia 14:1502-1508.

Borner C and Monney L (1999). Apoptosis without caspases: An inefficient molecular guillotine? Cell Death Differ 6:497507.

Chan SL and Mattson MP (1999). Caspase and calpain substrates: Roles in synaptic plasticity and cell death. J Neurosci Res 58:167-190.

Chi S, Kitanaka C, Noguchi K, Mochizuki T, Nagashima Y, Shirouzu M, Fujita H, Yoshida M, Chen W, Asai A, Himeno M, Yokoyama S, and Kuchino Y (1999). Oncogenic Ras triggers cell suicide through the activation of a caspase-independent cell death program in human cancer cells. Oncogene 18: 2281-2290.

Christensen JG, Goldsworthy TL, and Cattley RC (1999). Dysregulation of apoptosis by c-myc in transgenic hepato- 
cytes and effects of growth factors and nongenotoxic carcinogens. Mol Carcinog 25:273-284.

Columbano A (1995). Cell death: Current difficulties in discriminating apoptosis from necrosis in the context of pathological processes in vivo. J Cell Biochem 58:181-190.

Conzen SD, Gottlob K, Kandel ES, Khanduri P, Wagner AJ, O'Leary M, and Hay N (2000). Induction of cell cycle progression and acceleration of apoptosis are two separable functions of c-Myc: Transrepression correlates with acceleration of apoptosis. Mol Cell Biol 20:6008-6018.

Dang CV, Resar LM, Emison E, Kim S, Li Q, Prescott JE, Wonsey D, and Zeller K (1999). Function of the c-Myc oncogenic transcription factor. Exp Cell Res 253:63-77.

Daugas E, Nochy D, Ravagnan L, Loeffler M, Susin SA, Zamzami N, and Kroemer G (2000a). Apoptosis-inducing factor (AIF): A ubiquitous mitochondrial oxidoreductase involved in apoptosis. FEBS Lett 476:118-123.

Daugas E, Susin SA, Zamzami N, Ferri KF, Irinopoulou T, Larochette N, Prevost MC, Leber B, Andrews D, Penninger J, and Kroemer G (2000b). Mitochondrio-nuclear translocation of AIF in apoptosis and necrosis. FASEB J 14:729-739.

Duelli DM and Lazebnik YA (2000). Primary cells suppress oncogene-dependent apoptosis. Nat Cell Biol 2:859-862.

Elliott K, Ge K, Du W, and Prendergast GC (2000). The c-Myc-interacting adaptor protein Bin1 activates a caspaseindependent cell death program. Oncogene 19:4669-4684.

Evan GI, Wyllie AH, Gilbert CS, Littlewood TD, Land H, Brooks M, Waters CM, Penn LZ, and Hancock DC (1992). Induction of apoptosis in fibroblasts by c-myc protein. Cell 69:119-128.

Felsher DW and Bishop JM (1999). Reversible tumorigenesis by MYC in hematopoietic lineages. Mol Cell 4:199-207.

Fiers W, Beyaert R, Declercq W, and Vandenabeele P (1999). More than one way to die: Apoptosis, necrosis and reactive oxygen damage. Oncogene 18:7719-7730.

Fridman JS, Benedict MA, and Maybaum J (1999). bcl-X(S)induced cell death in 3T3 cells does not require or induce caspase activation. Cancer Res 59:5999-6004.

Fridman JS, Parsels J, Rehemtulla A, and Maybaum J (2001). Cytochrome c depletion upon expression of Bcl-XS. J Biol Chem 276:4205-4210.

Geske FJ, Monks J, Lehman L, and Fadok VA (2002). The role of the macrophage in apoptosis: Hunter, gatherer, and regulator. Int J Hematol 76:16-26.

Guo M and Hay BA (1999). Cell proliferation and apoptosis. Curr Opin Cell Biol 11:745-752.

Hirsch T, Marchetti P, Susin SA, Dallaporta B, Zamzami N, Marzo I, Geuskens M, and Kroemer G (1997). The apoptosisnecrosis paradox. Apoptogenic proteases activated after mitochondrial permeability transition determine the mode of cell death. Oncogene 15:1573-1581.

Hoffman B and Liebermann DA (1998). The proto-oncogene c-myc and apoptosis. Oncogene 17:3351-3357.

Hotti A, Jarvinen K, Siivola P, and Holtta E (2000). Caspases and mitochondria in c-Myc-induced apoptosis: Identification of ATM as a new target of caspases. Oncogene 19:23542362.

Hundley JE, Koester SK, Troyer DA, Hilsenbeck SG, Barrington RE, and Windle JJ (1997). Differential regulation of cell cycle characteristics and apoptosis in MMTV-myc and MMTV-ras mouse mammary tumors. Cancer Res 57:600603.

Jensen NA, Pedersen KM, Celis JE, and West MJ (1998). Failure of central nervous system myelination in MBP/c-myc transgenic mice: Evidence for c-myc cytotoxicity. Oncogene 16:2123-2129.

Johnson DE (2000). Noncaspase proteases in apoptosis. Leukemia 14:1695-1703.

Juin P, Hueber AO, Littlewood T, and Evan G (1999). c-Mycinduced sensitization to apoptosis is mediated through cytochrome c release. Genes Dev 13:1367-1381.

Kerr JF, Wyllie AH, and Currie AR (1972). Apoptosis: A basic biological phenomenon with wide-ranging implications in tissue kinetics. Br J Cancer 26:239-257.

Kolenko V, Uzzo RG, Bukowski R, Bander NH, Novick AC, Hsi ED, and Finke JH (1999). Dead or dying: Necrosis versus apoptosis in caspase-deficient human renal cell carcinoma. Cancer Res 59:2838-2842.

Kroemer G, Dallaporta B, and Resche-Rigon M (1998). The mitochondrial death/life regulator in apoptosis and necrosis. Annu Rev Physiol 60:619-642.

Levin S, Bucci TJ, Cohen SM, Fix AS, Hardisty JF, LeGrand EK, Maronpot RR, and Trump BF (1999). The nomenclature of cell death: Recommendations of an ad hoc Committee of the Society of Toxicologic Pathologists. Toxicol Pathol 27: 484-490.

Li K, Li Y, Shelton JM, Richardson JA, Spencer E, Chen ZJ, Wang X, and Williams RS (2000). Cytochrome c deficiency causes embryonic lethality and attenuates stress-induced apoptosis. Cell 101:389-399.

Liao DJ and Dickson RB (2000). c-Myc in breast cancer. Endocr Relat Cancer 7:143-164.

Liao DJ, Natarajan G, Deming SL, Jamerson MH, Johnson M, Chepko G, and Dickson RB (2000a). Cell cycle basis for the onset and progression of c-Myc-induced, TGFalphaenhanced mouse mammary gland carcinogenesis. Oncogene 19:1307-1317.

Liao DZ, Hou X, Bai S, Li SA, and Li JJ (2000b). Unusual deregulation of cell cycle components in early and frank estrogen-induced renal neoplasias in the Syrian hamster. Carcinogenesis 21:2167-2173.

Lockshin RA (1999). Commentary: The utility of apoptosis terminology. Toxicol Pathol 27:492-493.

Loeffler M and Kroemer G (2000). The mitochondrion in cell death control: Certainties and incognita. Exp Cell Res 256: 19-26.

Lorenzo HK, Susin SA, Penninger J, and Kroemer G (1999). Apoptosis inducing factor (AIF): A phylogenetically old, caspase-independent effector of cell death. Cell Death Differ 6:516-524.

Majno G and Joris I (1999). Commentary: On the misuse of the term "necrosis": A step in the right direction. Toxicol Pathol 27:494.

McCarthy NJ, Whyte MK, Gilbert CS, and Evan GI (1997). Inhibition of Ced-3/ICE-related proteases does not prevent cell death induced by oncogenes, DNA damage, or the Bcl-2 homologue Bak. J Cell Biol 136:215-227. 
McCormack SJ, Weaver Z, Deming S, Natarajan G, Torri J, Johnson MD, Liyanage M, Ried T, and Dickson RB (1998). $\mathrm{Myc} / \mathrm{p} 53$ interactions in transgenic mouse mammary development, tumorigenesis and chromosomal instability. Oncogene 16:2755-2766.

Messmer UK and Pfeilschifter J (2000). New insights into the mechanism for clearance of apoptotic cells. Bioessays 22: $878-881$.

Nicotera P, Leist M, and Ferrando-May E (1999). Apoptosis and necrosis: Different execution of the same death. Biochem Soc Symp 66:69-73.

Osthus RC, Shim H, Kim S, Li Q, Reddy R, Mukherjee M, Xu Y, Wonsey D, Lee LA, and Dang CV (2000). Deregulation of glucose transporter 1 and glycolytic gene expression by c-Myc. J Biol Chem 275:21797-21800.

Packham G and Cleveland JL (1995). c-Myc and apoptosis. Biochim Biophys Acta 1242:11-28.

Pelengaris S, Rudolph B, and Littlewood T (2000). Action of Myc in vivo: proliferation and apoptosis. Curr Opin Genet Dev 10:100-105.

Prendergast GC (1999). Mechanisms of apoptosis by c-Myc. Oncogene 18:2967-2987.

Sanders S and Thorgeirsson SS (1999). Phenobarbital promotes liver growth in c-myc/TGF-alpha transgenic mice by inducing hypertrophy and inhibiting apoptosis. Carcinogenesis 20:41-49.

Sandgren EP, Quaife CJ, Pinkert CA, Palmiter RD, and Brinster RL (1989). Oncogene-induced liver neoplasia in transgenic mice. Oncogene 4:715-724.
Savill J and Fadok V (2000). Corpse clearance defines the meaning of cell death. Nature 407:784-788.

Shi Y, Glynn JM, Guilbert LJ, Cotter TG, Bissonnette RP, and Green DR (1992). Role for c-myc in activation-induced apoptotic cell death in T cell hybridomas. Science 257:212-214.

Shim H, Dolde C, Lewis BC, Wu CS, Dang G, Jungmann RA, Dalla-Favera R, and Dang CV (1997). c-Myc transactivation of LDH-A: implications for tumor metabolism and growth. Proc Natl Acad Sci USA 94:6658-6663.

Shim H, Chun YS, Lewis BC, and Dang CV (1998a). A unique glucose-dependent apoptotic pathway induced by c-Myc. Proc Natl Acad Sci USA 95:1511-1516.

Skulachev VP (1998). Cytochrome $c$ in the apoptotic and antioxidant cascades. FEBS Lett 423:275-280.

Stewart TA, Pattengale PK, and Leder P (1984). Spontaneous mammary adenocarcinomas in transgenic mice that carry and express MTV/myc fusion genes. Cell 38:627-637.

Thompson EB (1998). The many roles of c-Myc in apoptosis. Annu Rev Physiol 60:575-600.

Trudel M, Lanoix J, Barisoni L, Blouin MJ, Desforges M, L'Italien C, and D'Agati V (1997). C-myc-induced apoptosis in polycystic kidney disease is $\mathrm{Bcl}-2$ and p53 independent. $\mathrm{J}$ Exp Med 186:1873-1884.

Vier J, Linsinger G, and Hacker G (1999). Cytochrome c is dispensable for fas-induced caspase activation and apoptosis. Biochem Biophys Res Commun 261:71-78. 